\title{
On the Regularity of the Partial $O *$-Algebras Generated by a Closed Symmetric Operator
}

By

\author{
Jean-Pierre AntoIne*, Atsushi InOuE** and Camillo Trapani***
}

\begin{abstract}
Let be given a dense domain $\mathscr{D}$ in a Hilbert space and a closed symmetric operator $T$ with domain containing $\mathscr{D}$. Then the restriction of $T$ to $\mathscr{D}$ generates (algebraically) two partial $*$ algebras of closable operators (called weak and strong), possibly nonabelian and nonassociative. We characterize them completely. In particular, we examine under what conditions they are regular, that is, consist of polynomials only, and standard. Simple differential operators provide concrete examples of all the pathologies allowed by the abstract theory.
\end{abstract}

\section{§1. Introduction}

In two recent papers $[1,2]$, we have started a systematic investigation of partial *-algebras of closable operators, for short, partial $O^{*}$-algebras. By this we mean a collection of closable operators in a Hilbert space, defined on a common dense domain, which is not necessarily invariant, and equipped with appropriate operations of addition and (partial) multiplication. The resulting algebraic structure is a generalization of *-algebras of bounded operators $\left(W^{*}\right.$ -

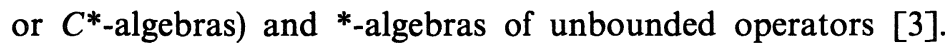

One of the motivations for that work, as well as our previous work with Karwowski $[4,5]$ and Mathot [6,7], is the formulation of quantum theories, where the algebraic approach has proven to be both elegant and efficient. We have argued previously [1] that partial $O^{*}$-algebras are a natural tool in this context, and there is no need of repeating those arguments here. Instead we

Communicated by H. Araki, July 19, 1991. Revised November 13, 1991.

1991 Mathematics Subject Classification: 47D40

* Institut de Physique Théorique, Université Catholique de Louvain, B-1348-Louvain-la-Neuve, Belgium

** Department of Applied Mathematics, Fukuoka University, Fukuoka 814, Japan

*** Instituto di Fisica dell'Università di Palermo, I-90123-Palermo, Italy 
would like to focus on one particular aspect, that of a "complete set of commuting observables" (CSCO), which plays a basic role in Dirac's formulation of Quantum Mechanics. In the Hilbert space framework, an observable is represented by a self-adjoint operator, thus a CSCO is a maximal set of (strongly) commuting self-adjoint operators. In algebraic language, a CSCO is nothing but a maximal abelian von Neumann algebra. The concept was later extended to unbounded operators, using the so-called $V^{*}$-algebras $[8,9]$.

But now two remarks are in order. First, one ought to extend the concept of CSCO to the case of a partial $O^{*}$-algebra. Second, it may be too restrictive to consider only self-adjoint observables. Indeed recent developments in the description of quantum mechanical measurements and their repeatability give an increasingly important role to positive operator valued (POV) measures. And whereas projection valued measures generate self-adjoint operators, the operator generated by a POV-measure, if it exists at all, is in general only symmetric [10-13].

The aim of the present paper is to provide an answer to these two questions. More precisely, we want to investigate the case of a single observable, represented by a closed symmetric operator: What kind of structure does it generate in the context of a partial $O^{*}$-algebra? ('Generate' is taken here in the naive sense, by sums and products, not by considering bicommutants, as is done in the algebraic formulation, both with von Neumann algebras and with $V^{*}$ algebras $[8,9]$ ). Actually there are two structures to examine, since there are two partial multiplications, the weak one and the strong one. The answer is expected to be tricky, since both partial multiplications are in general nonassociative. And indeed we will find that quite often the symmetric operator generates a partial $O^{*}$-algebra which is neither abelian, nor associative!

The paper is organized as follows. We begin by recalling in Section 2 the basic definitions about partial $(O)^{*}$-algebras. Then, in Section 3, we fix a closed symmetric operator $T$ in a Hilbert space $\mathscr{H}$ and a core $\mathscr{D}$ for $T$, and we study the partial $O^{*}$-algebras on $\mathscr{D}$ generated by the restriction of $T$ to $\mathscr{D}$, denoted by $\mathbb{T}^{[1]}$; these are called $\mathfrak{M}_{W}\left(T^{[1]}\right)$ and $\mathfrak{M}_{S}\left(T^{[1]}\right)$, for the weak and the strong partial multiplication, respectively. These objects have been introduced in [1], and their structure determined. In particular, it has been shown that, in general, both partial $O^{*}$-algebras possess a regular part, consisting of polynomials in $T^{[1]}$ (with respect to the weak or the strong partial multiplication), and a singular part, the elements of which cannot in general be written as a polynomial. Here we focus our attention to the question of regularity of the two partial $O^{*}$-algebras, i.e. we look for conditions under which their singular part is empty. In this case, they are of course abelian and associative. As expected, the answer lies in the "naturalness" of the domain $\mathscr{D}$ with respect to $\mathbb{T}$. For instance, a sufficient condition is that $T$ be maximal and that $\mathscr{D}$ contain all the $C^{\infty}$-vectors of $T$. 
In Section 4, we turn to the question of standardness of the two partial $O^{*}$-algebras $\mathfrak{M}_{W}\left(T^{[1]}\right)$ and $\mathfrak{M}_{S}\left(T^{[1]}\right)$. This notion, which generalizes that introduced by Powers [14] in the case of abelian $O^{*}$-algebras, was also defined in [1]. Some results in this direction were already obtained in that paper, and we shall extend them here. Finally, in Section 5, we exhibit some explicit examples and counterexamples, all of them differential operators, which show that all the pathologies described in the general theory do in fact occur in practice.

It is interesting to note in this respect that such a bad behavior may persist even if the operator $T$ is self-adjoint: here too, regularity follows only if the domain $\mathscr{D}$ is "natural". This is in fact the general lesson of the present study, and it is entirely in agreement with the philosophy developed in all quantum mechanical formalisms based on the notion of "selected" observables $[5,8$, and references quoted therein].

\section{§ 2. Partial $O^{*}$-algebras}

In order to make the paper self-contained, we shall repeat here the essential definitions. For further details, we refer the reader to $[1,6]$.

A partial *-algebra is a complex vector space $\mathfrak{A}$ with an involution $x \mapsto x^{*}$ (i.e. $\left.(x+\lambda y)^{*}=x^{*}+\bar{\lambda} y^{*}, x^{* *}=x, x, y \in \mathfrak{U}, \lambda \in \mathbb{C}\right)$ and a subset $\Gamma \subset \mathfrak{U} \times \mathfrak{U}$ such that:

(i) $(x, y) \in \Gamma$ iff $\left(y^{*}, x^{*}\right) \in \Gamma$;

(ii) if $(x, y) \in \Gamma$ and $(x, z) \in \Gamma$, then $(x, \lambda y+\mu z) \in \Gamma$ for all $\lambda, \mu \in \mathbb{C}$;

(iii) whenever $(x, y) \in \Gamma$, there exists an element $x \cdot y \in \mathfrak{Q}$ with the usual properties of the multiplication:

$$
x \cdot(y+\lambda z)=x \cdot y+\lambda(x . z) \text { and }(x, y)^{*}=y^{*} \cdot x^{*}, \text { for }(x, y),(x, z) \in \Gamma \text { and } \lambda \in \mathbb{C} .
$$

An element $e$ of $\mathfrak{A}$ is said to be a unit if $e^{*}=e,(e, x) \in \Gamma$ and $e x=x e=x$ for every $x \in \mathfrak{U}$.

Whenever $(x, y) \in \Gamma$, we simply say that the product $x . y$ exists. Notice the analogy with the notion of groupoid.

We emphasize that the multiplication is not required to be associative. This makes the structure of abelian partial *-algebras much trickier than usual, as we shall see below. On the other hand, abelianness is defined in the natural way: A partial *-algebra $\mathfrak{A}$ is said to be abelian, or commutative, if the following conditions hold:

(i) $(x, y) \in \Gamma \Leftrightarrow(y, x) \in \Gamma, \quad x, y \in \mathfrak{A}$;

(ii) $x \cdot y=y \cdot x, \quad \forall(x, y) \in \Gamma$.

To fix ideas, we give a simple example, namely that of partial *-algebras of polynomials. Let $\mathfrak{P}(z)$ be the set of all complex polynomials of arbitrary 
degree in the real variable $z \cdot \mathfrak{P}(z)$ is an abelian *-algebra (where $*$ is understood to be the complex conjugation), but it contains plenty of abelian partial *algebras. Denote by $\mathfrak{P}_{r}(z)$ the set of all polynomials of degree at most $r$ (by $\delta p$ we mean the degree of the polynomial $p$ ):

$$
\mathfrak{P}_{r}(z)=\{p(z) \in \mathfrak{P}(z) \mid \delta p \leq r\} .
$$

It is readily checked that $\mathfrak{P}_{r}(z)$ is an abelian partial *-algebra when we take for $\Gamma$ the following set

$$
\Gamma=\left\{(p, q) \in \mathfrak{P}_{r}(z) \times \mathfrak{P}_{r}(z) \mid \delta p+\delta q \leq r\right\} .
$$

Actually this is the basic example: a partial *-algebra will be called regular whenever it is isomorphic to a usual polynomial partial *-algebra $\mathfrak{P}_{r}$ for some $r \leq \infty$.

One of the simplest examples of abelian *-algebras is that generated by a single hermitian element of a nonabelian *-algebra. If $\mathfrak{U}$ is a *-algebra and $x=x^{*} \in \mathfrak{U}$, the *-algebra $\mathfrak{M}(x)$ generated by $x$ is well-defined as the intersection of all *-subalgebras of $\mathfrak{A}$ containing $x$, and it consists of all polynomials in $x$. But, for partial *-algebras, the situation is more involved, since (i) an element $x$ cannot necessarily be multiplied by itself; (ii) if they are defined, we may have several $n^{\text {th }}$ powers of $x$, because of the failure of associativity. So abelianness may fail in general!

From this we gather that much care has to be exercised in the definition of successive powers of a given element. Instead of examining the situation in the abstract setting, we will concentrate in this paper on the case of a closed symmetric operator on a Hilbert space. The full discussion may be found in [6].

Let $\mathscr{H}$ be a Hilbert space and $\mathscr{D}$ a fixed dense subspace of $\mathscr{H}$. We denote by $\mathscr{L}^{+}(\mathscr{D}, \mathscr{H})$ the set of all (closable) linear operators $X$ such that $\mathscr{D}(X)=\mathscr{D}, \mathscr{D}\left(X^{*}\right) \supset \mathscr{D}$. The set $\mathscr{L}^{+}(\mathscr{D}, \mathscr{H})$ is a partial *-algebra with unit, denoted $\mathscr{L}_{w}^{+}(\mathscr{D}, \mathscr{H})$, with respect to the following operations: the usual sum $X_{1}+X_{2}$, the scalar multiplication $\lambda X$, the involution $X \mapsto X^{+} \equiv X^{*}\lceil\mathscr{D}$ and the weak partial multiplication $X_{1} \square X_{2}=X_{1}{ }^{+*} X_{2}$, defined whenever $X_{2} \mathscr{D} \subset$ $\mathscr{D}\left(X_{1}{ }^{+*}\right)$ and $X_{1}{ }^{+} \mathscr{D} \subset \mathscr{D}\left(X_{2}^{*}\right)$. Then a weak partial $O^{*}$-algebra on $\mathscr{D}$ is a partial *-subalgebra $\mathfrak{M}$ of $\mathscr{L}_{w}^{+}(\mathscr{D}, \mathscr{H})$; that is, $\mathfrak{M}$ is a subspace of $\mathscr{L}^{+}(\mathscr{D}, \mathscr{H})$ such that $X^{+} \in \mathfrak{M}$ for each $X \in \mathfrak{M}$, and $X_{1} \square X_{2} \in \mathfrak{M}$ whenever $X_{1}, X_{2} \in \mathfrak{M}$ and $X_{1} \square X_{2}$ exists. (Remark: in the previous literature, these were called partial Op*-algebras; here we follow the terminology of Schmüdgen [3]).

On $\mathscr{L}^{+}(\mathscr{D}, \mathscr{H})$ we also consider the strong partial multiplication: $X_{1} \bullet X_{2}=$ $\overline{X_{1}} X_{2}$, defined whenever $X_{2} \mathscr{D} \subset \mathscr{D}\left(\overline{X_{1}}\right)$ and $X_{1}{ }^{+} \mathscr{D} \subset \mathscr{D}\left(\overline{X_{2}{ }^{+}}\right)$. Equipped with this partial multiplication, $\mathscr{L}^{+}(\mathscr{D}, \mathscr{H})$ is denoted by $\mathscr{L}_{s}^{+}(\mathscr{D}, \mathscr{H})$. However $\mathscr{L}_{s}^{+}(\mathscr{D}, \mathscr{H})$ is in general not a partial *-algebra, because the strong partial multiplication may fail to be distributive with respect to the addition $[4,6]$. 
Thus we define a strong partial $O^{*}$-algebra as a subspace of $\mathscr{L}_{s}^{+}(\mathscr{D}, \mathscr{H})$ which is stable under all operations and in which, in addition, the distributive law holds.

A remarkable subset of $\mathscr{L}^{+}(\mathscr{D}, \mathscr{H})$ is the *-algebra $\mathscr{L}^{+}(\mathscr{D})=\left\{A \in \mathscr{L}^{+}(\mathscr{D}, \mathscr{H})\right.$; $A \mathscr{D} \subset \mathscr{D}$ and $\left.A^{+} \mathscr{D} \subset \mathscr{D}\right\}$. A *-subalgebra of $\mathscr{L}^{+}(\mathscr{D})$ containing the identity operator is called an $O^{*}$-algebra on $\mathscr{D}[3,15-17]$.

As usual [14], a (partial) $O^{*}$-algebra $\mathfrak{M}$ is called standard if every operator $X \in \mathfrak{M}$ satisfies the condition $\overline{X^{+}}=X^{*}$. Notice that in this case we don't have to distinguish between strong and weak multiplication, since the two notions now coincide.

Let $\mathfrak{N}$ be a ${ }^{+}$-invariant subset of $\mathscr{L}^{+}(\mathscr{D}, \mathscr{H})$. Then there is a minimal weak partial $O^{*}$-algebra on $\mathscr{D}$ containing $\mathfrak{N}$, that we denote by $\mathfrak{M}_{w}[\mathfrak{N}]$, defined simply as the intersection of all weak partial $O^{*}$-algebras containing $\mathfrak{N}$. In the strong case, the corresponding object $\mathfrak{M}_{s}[\mathfrak{N}]$, defined in the same way, does not necessarily exist, since $\mathscr{L}_{s}^{+}(\mathscr{D}, \mathscr{H})$ may fail to be a partial *-algebra. However, as shown in [1], $\mathfrak{M}_{s}[\mathfrak{N}]$ does exist if $\mathfrak{N}=\{T \uparrow \mathscr{D}\}$, where $T$ is a closed symmetric operator in $\mathscr{H}$. The two partial $O^{*}$-algebras $\mathfrak{M}_{w}(T \uparrow \mathscr{D})$ and $\mathfrak{M}_{s}(T \nmid \mathscr{D})$ form the subject matter of the present paper.

We recall some additional terminology about partial $O^{*}$-algebras $[1,4,6]$. Given a ${ }^{+}$-invariant subset $\mathfrak{N}$ of $\mathscr{L}^{+}(\mathscr{D}, \mathscr{H})$, define the two domains:

$$
\begin{gathered}
\hat{\mathscr{D}}(\mathfrak{N})=\bigcap_{X \in \mathfrak{N}} \mathscr{D}(\bar{X}), \\
\mathscr{D}^{*}(\mathfrak{N})=\bigcap_{\boldsymbol{X} \in \mathfrak{N}} \mathscr{D}\left(X^{*}\right),
\end{gathered}
$$

so that

$$
\mathscr{D} \subset \hat{\mathscr{D}}(\mathfrak{R}) \subset \mathscr{D}^{*}(\mathfrak{R}) .
$$

Then $\mathfrak{R}$ is said to be fully closed if $\hat{\mathscr{D}}(\mathfrak{N})=\mathscr{D}$, essentially self-adjoint if $\mathscr{D}^{*}(\mathfrak{N})=$ $\hat{\mathscr{D}}(\mathfrak{N})$ and self-adjoint if $\mathscr{D}^{*}(\mathfrak{N})=\mathscr{D}$.

Finally we will need the two closable operators:

$$
\begin{aligned}
\hat{\imath}(X) & =\bar{X} \uparrow \hat{\mathscr{D}}(\mathfrak{N}), \quad X \in \mathfrak{N}, \\
\imath^{*}(X) & =X^{+*} \uparrow \mathscr{D}^{*}(\mathfrak{N}), \quad X \in \mathfrak{N} .
\end{aligned}
$$

In particular, the fully closure of $\mathfrak{N}$ is the set:

$$
\hat{\mathfrak{N}} \equiv \hat{\imath}(\mathfrak{N})=\{\hat{\imath}(X) ; X \in \mathfrak{N}\},
$$

which is clearly a subspace of $\mathscr{L}^{+}(\hat{\mathscr{D}}(\mathfrak{N}), \mathscr{H})$.

\section{§3. Regularity of the Partial $O^{*}$-algebras $\mathfrak{D}_{w}\left(T^{[1]}\right)$ and $\mathfrak{M}_{s}\left(T^{[1]}\right)$}

Throughout this section, $T$ is a closed symmetric unbounded operator in $\mathscr{H}, \mathscr{D}$ a core for $T, T^{[1]}=T \uparrow \mathscr{D}$ and $n$ the largest number among all $k \in \mathbb{N} \cup$ 
$\{\infty\}$ such that $\mathscr{D} \subset \mathscr{D}\left(T^{k}\right)$ (for $n=\infty$, we mean $\mathscr{D}\left(T^{\infty}\right) \equiv \mathscr{D}^{\infty}(T)=\bigcap_{k=1}^{\infty} \mathscr{D}\left(T^{k}\right)$ ). We want to study the weak and strong partial $O^{*}$-algebras on $\mathscr{D}$ generated by $T^{[1]}$. We will denote them by $\mathfrak{M}_{w}\left(T^{[1]}\right)$ and $\mathfrak{M}_{s}\left(T^{[1]}\right)$, respectively. We define:

$$
\begin{aligned}
T^{[k]} & =T^{k} \uparrow \mathscr{D}, \quad 1 \leq k \leq n ; \\
\mathfrak{B}_{r}\left(T^{[1]}\right) & =\left\{\sum_{k=0}^{r} \alpha_{k} T^{[k]} ; \alpha_{k} \in \mathbb{C}, k=0,1,2, \ldots, r\right\}, 1 \leq r \leq n, \text { if } n \in \mathbb{N} ; \\
\mathfrak{P}\left(T^{[1]}\right) & \equiv \mathfrak{P}_{\infty}\left(T^{[1]}\right)=\left\{\sum_{k=0}^{r} \alpha_{k} T^{[k]} ; \alpha_{k} \in \mathbb{C}, k=0,1,2, \ldots, r ; r \in \mathbb{N}\right\}, \text { if } n=\infty .
\end{aligned}
$$

As it is well-known [1], the norms $\|\cdot\|_{P(T)}$ and $\|\cdot\|_{T^{r}}$ are equivalent for every complex polynomial $P$ of degree $r$ (here, as usual, $\|\cdot\|_{A}$ denotes the graph norm of an operator $A$ : $\|\xi\|_{A}^{2}=\|A \xi\|^{2}+\|\xi\|^{2}, \forall \xi \in \mathscr{D}(A)$ ), and a subspace $\mathscr{D} \subset$ $\mathscr{D}\left(T^{r}\right)$ is a core for $T^{r}$ iff it is a core for every complex polynomial $P(T)$ of degree $r$. Hence it suffices to study the powers of $T$ for controlling arbitrary polynomials in $T$. So we have to define properly the successive powers of $T^{[1]}$, both weak and strong.

It is easy to see that, when $n \in \mathbb{N}, T^{[k]} \square T^{[m-k]}$ exists and equals $T^{[m]}$ for each $m \leq n$ and each $k<m$. Thus, for each $m=1,2, \ldots, n, \mathbb{T}^{[m]}$ is the weak $m^{\text {th }}$ power $T^{[1]} \square \cdots \square T^{[1]}\left(m\right.$ times) of $T^{[1]}$ (hence the notation is consistent). Higher weak powers of $T^{[1]}$ are now defined recursively. If all products $T^{[k]} \square T^{[m]}$ exist for each pair $k, m \in \mathbb{N}$ with $k+m=n+1$ and they coincide, we say that the weak $(n+1)^{\text {th }}$ power of $T^{[1]}$ is defined and we denote it by $T^{[n+1]}$. Successive higher powers $T^{[n+2]}, T^{[n+3]}, \ldots$ may be defined in the same way, if the corresponding conditions hold.

For strong powers, the situation changes drastically. Let $m \leq n$. Then, if all products $T^{[k]} T^{[m-k]}$ exist for $k=1, \ldots m$ and they coincide, we say that the strong $m^{\text {th }}$ power of $T^{[1]}$ is defined; we denote it by $T^{(m)}$, although it coincides with $T^{[m]}$. But now the process stops at $m=n$, no higher strong power may be defined. Indeed, it is easy to see that, if $T^{[k]} \odot T^{[m]}$ exists for $k, m<n$, then $k+m \leq n$.

In order to visualize the behaviour of $T^{[1]}$ under the two partial multiplications, we denote by $l_{w} \equiv l_{w}\left(T^{[1]}\right)$ the largest number in $\mathbb{N} \cup\{\infty\}$ such that all the weak $k^{\text {th }}$ powers $T^{[k]}, 1 \leq k \leq l_{w}$, of $T^{[1]}$ are defined, and call it the weak length of $T^{[1]}$. The strong length $l_{s} \equiv l_{s}\left(T^{[1]}\right)$ is defined in an analogous way. Thus the discussion above may be summarized by the inequalities $n \leq l_{w} \leq \infty$, $1 \leq l_{s} \leq n$.

In that language, the structure of the partial $O^{*}$-algebras $\mathfrak{D}_{w}\left(T^{[1]}\right)$ and $\mathfrak{M}_{s}\left(T^{[1]}\right)$ is described by the following theorem, obtained in [1]:

Theorem 3.1. Let $n$ be the largest number among all $k \in \mathbb{N} \cup\{\infty\}$ such that $\mathscr{D} \subset \mathscr{D}\left(T^{k}\right)$ and $m$ be the largest number among all $k \in \mathbb{N} \cup\{0\} \cup\{\infty\}$ such that $T^{n} \mathscr{D} \subset \mathscr{D}\left(T^{* k}\right)$. Then the following statements hold: 
(1) $1 \leq l_{s} \leq n \leq \min (m, n)+n \leq l_{w} \leq m+n$.

In particular, if $T$ is self-adjoint, then $l_{w}=n$.

(2) If $n=\infty$, that is, $\mathscr{D} \subset \mathscr{D}^{\infty}(T)$, then $l_{w}=\infty$ and $\mathfrak{M}_{w}\left(T^{[1]}\right)=\mathfrak{B}\left(T^{[1]}\right)$, thus it is abelian and associative.

(3) If $n<\infty$, then

$$
\mathfrak{M}_{w}\left(T^{[1]}\right)=\mathfrak{R}_{w}\left(T^{[1]}\right)+\mathfrak{S}_{w}\left(T^{[1]}\right),
$$

where $\mathfrak{R}_{w}\left(T^{[1]}\right)$, the regular part of $\mathfrak{M}_{w}\left(T^{[1]}\right)$, equals the polynomial algebra $\mathfrak{P}_{l_{w}}\left(T^{[1]}\right)$, and $\mathfrak{S}_{w}\left(T^{[1]}\right)$, the singular part, consists of elements which cannot be represented as polynomials. Then $\mathfrak{M}_{w}\left(T^{[1]}\right)$ need not be abelian, nor associative.

(4) $\mathfrak{M}_{s}\left(T^{[1]}\right)$ always exists and may be written as

$$
\mathfrak{M}_{s}\left(T^{[1]}\right)=\mathfrak{R}_{s}\left(T^{[1]}\right)+\mathfrak{S}_{s}\left(T^{[1]}\right),
$$

where $\mathfrak{R}_{s}\left(T^{[1]}\right)=\left\{\sum_{k=0}^{l_{s}} \alpha_{k} T^{(k)} ; \alpha_{k} \in \mathbb{C}\left(k=1,2, \ldots, l_{s}\right)\right\}$ is the regular part, and $\mathfrak{S}_{s}\left(T^{[1]}\right)$, the singular part, consists of polynomials of degree at most $n . \mathfrak{M}_{s}\left(T^{[1]}\right)$ is in general neither abelian, nor associative.

(5) $\mathfrak{M}_{s}\left(T^{[1]}\right) \subset \mathfrak{P}_{n}\left(T^{[1]}\right) \subset \mathfrak{M}_{w}\left(T^{[1]}\right)$.

In this Section we investigate under what conditions $\mathfrak{M}_{w}\left(T^{[1]}\right)$ and $\mathfrak{M}_{s}\left(T^{[1]}\right)$ are regular, that is, coincide with a polynomial algebra $\mathfrak{P}_{r}\left(T^{[1]}\right)=\left\{\sum_{k=0}^{r} \alpha_{k} T^{[k]}\right.$; $\left.\alpha_{k} \in \mathbb{C}, k=0,1,2, \ldots, r\right\}$, in which case they are abelian and associative.

Proposition 3.2. Let $n$ be the largest number among all $k \in \mathbb{N} \cup\{\infty\}$ such that $\mathscr{D} \subset \mathscr{D}\left(T^{k}\right)$. Suppose $\mathscr{D}$ is a core for all $T^{k}, 1 \leq k \leq n$. Then $l_{s}\left(T^{[1]}\right)=n$ and $\mathfrak{M}_{s}\left(T^{[1]}\right)=\mathfrak{P}_{n}\left(T^{[1]}\right)$, but $\mathfrak{P}_{n}\left(T^{[1]}\right) \subsetneq \mathfrak{M}_{w}\left(T^{[1]}\right)$ in general.

Proof. Since $\mathscr{D}$ is a core for every polynomial of degree at most $n$ ([3], Corollary 1.2), we see that $l_{s}\left(T^{[1]}\right)=n$ and $\mathfrak{M}_{s}\left(T^{[1]}\right)=\mathfrak{P}_{n}\left(T^{[1]}\right)$. Examples where $\mathfrak{P}_{n}\left(T^{[1]}\right)$ is different from $\mathfrak{M}_{w}\left(T^{[1]}\right)$ will be given in Corollary 3.7 and Examples 5.2, 5.3 below.

We shall first examine the regularity of $\mathfrak{M}_{s}\left(T^{[1]}\right)$ and $\mathfrak{M}_{w}\left(T^{[1]}\right)$ when $n=\infty$. The following result is immediate.

Proposition 3.3. Let $\mathscr{D}$ be a dense subspace of $\mathscr{H}$ contained in $\mathscr{D}^{\infty}(T)$ and $T^{[1]}=T \uparrow \mathscr{D}$. Then the following statements hold.

(1) $l_{w}\left(T^{[1]}\right)=\infty$ and $\mathfrak{M}_{w}\left(T^{[1]}\right)=\mathfrak{P}\left(T^{[1]}\right)$.

(2) Suppose $\mathscr{D}$ is a core for $T^{k}, \forall k \in \mathbb{N}$. Then

$$
\begin{gathered}
l_{w}\left(T^{[1]}\right)=l_{s}\left(T^{[1]}\right)=\infty, \\
\mathfrak{M}_{w}\left(T^{[1]}\right)=\mathfrak{M}_{s}\left(T^{[1]}\right)=\mathfrak{B}\left(T^{[1]}\right),
\end{gathered}
$$

and its full closure is a closed $O^{*}$-algebra on $\mathscr{D}^{\infty}(T)$.

We turn now to the case $n<\infty$. 
Lemma 3.4. (1) Suppose $\mathscr{D}^{\infty}(T)$ is a core for all $T^{k}, k \in \mathbb{N}$. Then

$$
\mathscr{D}\left(T^{* k}\right)=\bigcap_{i=1}^{k} \mathscr{D}\left(T^{i *}\right), \quad \forall k \in \mathbb{N} .
$$

(2) Suppose $T$ is maximal symmetric or that $T \mathscr{D}^{\infty}(T)$ is a core for all $T^{k}$, $k \in \mathbb{N}$. Then

$$
\mathscr{D}\left(T^{* k}\right)=\mathscr{D}\left(T^{k *}\right), \quad \forall k \in \mathbb{N} .
$$

Proof. (1) Obvious.

(2) Suppose $T$ is maximal symmetric, so that $\rho(T) \neq \varnothing$. By Theorem 1.9 of [18], this implies that $\mathscr{D}^{\infty}(T)$ is a core for all $T^{k}, k \in \mathbb{N}$. Let $r_{0} \in \rho(T)$. We show that $\mathscr{D}\left(T^{k *}\right) \subset \mathscr{D}\left(\left(T-r_{0} 1\right)^{* k}\right)$. This is clear for $k=1$. Let now $k>1$. For every $\eta \in \mathscr{D}\left(T^{k *}\right)$ and $\xi \in \mathscr{D}^{\infty}(T)$, we have

$$
\left(T^{k} \xi \mid \eta\right)=\left(\xi \mid T^{k *} \eta\right)
$$

Since $\left(T-r_{0} 1\right)^{1-k} \xi \in \mathscr{D}^{\infty}(T)$ and $T^{k}=\alpha_{0} 1+\alpha_{1}\left(T-r_{0} 1\right)+\cdots+\left(T-r_{0} 1\right)^{k}$ for some $\alpha_{0}, \ldots, \alpha_{k-1} \in \mathbb{C}$, we have by (3.1)

$$
\begin{aligned}
& \left(\xi \mid\left\{\left(T-r_{0} 1\right)^{1-k}\right\}^{*} T^{k *} \eta\right) \\
& \quad=\left(T^{k}\left(T-r_{0} 1\right)^{1-k} \xi \mid \eta\right) \\
& \quad=\left(\left(T-r_{0} 1\right) \xi \mid \eta\right)+\left(\xi \mid \overline{\alpha_{k-1}} \eta\right)+\cdots+\left(\xi \mid \overline{\alpha_{0}}\left\{\left(T-r_{0} 1\right)^{1-k}\right\}^{*} \eta\right) .
\end{aligned}
$$

Hence we get

$$
\left(\left(T-r_{0} 1\right) \xi \mid \eta\right)=\left(\xi \mid \zeta_{1}\right)
$$

for some $\zeta_{1} \in \mathscr{H}$, and therefore, since $\mathscr{D}^{\infty}(T)$ is a core for $\left(T-r_{0} 1\right)$,

$$
\eta \in \mathscr{D}\left(\left(T-r_{0} 1\right)^{*}\right) \text {. }
$$

In the same way, since $\left(T-r_{0} 1\right)^{2-k} \xi \in \mathscr{D}^{\infty}(T)$, we have, by (3.1) and (3.2),

$$
\begin{aligned}
& \left(\xi \mid\left\{\left(T-r_{0} 1\right)^{2-k}\right\}^{*} T^{k *} \eta\right) \\
& \quad=\left(T^{k}\left(T-r_{0} 1\right)^{2-k} \xi \mid \eta\right) \\
& \quad=\left(\left(T-r_{0} 1\right) \xi \mid\left(T-r_{0} 1\right)^{*} \eta\right)+\left(\xi \mid \overline{\alpha_{k-1}}\left(T-r_{0} 1\right)^{*} \eta\right)+\cdots+\left(\xi \mid \overline{\alpha_{0}}\left\{\left(T-r_{0} 1\right)^{2-k}\right\}^{*} \eta\right),
\end{aligned}
$$

and so

$$
\left(\left(T-r_{0} 1\right) \xi \mid\left(T-r_{0} 1\right)^{*} \eta\right)=\left(\xi \mid \zeta_{2}\right)
$$

for some $\zeta_{2} \in \mathscr{H}$. Since $\mathscr{D}^{\infty}(T)$ is a core for $\left(T-r_{0} 1\right)^{2}$, we get $\eta \in \mathscr{D}\left(\left(T-r_{0} 1\right)^{* 2}\right)$. Repeating the argument, we obtain $\eta \in \mathscr{D}\left(\left(T-r_{0} 1\right)^{* k}\right)$. By a similar reasoning we prove $\mathscr{D}\left(\left(T-r_{0} 1\right)^{k *}\right) \subset \mathscr{D}\left(T^{* k}\right)$. Thus we have finally

$$
\mathscr{D}\left(T^{* k}\right) \subset \mathscr{D}\left(T^{k *}\right) \subset \mathscr{D}\left(\left(T-r_{0} 1\right)^{* k}\right) \subset \mathscr{D}\left(\left(T-r_{0} 1\right)^{k *}\right) \subset \mathscr{D}\left(T^{* k}\right),
$$

which implies the statement. 
Suppose now that $T \mathscr{D}^{\infty}(T)$ is a core for all $T^{k}, k \in \mathbb{N}$. It is clear that $\mathscr{D}\left(T^{* k}\right) \subset \mathscr{D}\left(T^{k *}\right), \forall k \in \mathbb{N}$. By induction we prove the converse inclusion:

$$
\mathscr{D}\left(T^{* k}\right) \supset \mathscr{D}\left(T^{k *}\right), \quad \forall k \in \mathbb{N} .
$$

This is clear for $k=1$. Suppose (3.3) holds for some $k \in \mathbb{N}$. Take any vector $\eta \in \mathscr{D}\left(\left(T^{k+1}\right)^{*}\right)$. Then we have

$$
\left(T^{k}(T \xi) \mid \eta\right)=\left(T^{k+1} \xi \mid \eta\right)=\left(\xi \mid\left(T^{k+1}\right)^{*} \eta\right), \quad \text { for all } \xi \in \mathscr{D}^{\infty}(T) .
$$

Since $T \mathscr{D}^{\infty}(T)$ is a core for $T^{k}$, we have $\eta \in \mathscr{D}\left(T^{k *}\right)$, which equals $\mathscr{D}\left(T^{* k}\right)$ by assumption. Hence we get, by (3.4),

$$
\left(T \xi \mid T^{* k} \eta\right)=\left(\xi \mid\left(T^{k+1}\right)^{*} \eta\right)
$$

for all $\xi \in \mathscr{D}^{\infty}(T)$. Since $\mathscr{D}^{\infty}(T)$ is a core for $T$, we have $T^{* k} \eta \in \mathscr{D}\left(T^{*}\right)$, and therefore $\eta \in \mathscr{D}\left(\left(T^{*}\right)^{k+1}\right)$.

Theorem 3.5. Let $T$ be a closed symmetric operator in $\mathscr{H}, \mathscr{D}$ a dense subspace of $\mathscr{H}$ containing $\mathscr{D}^{\infty}(T)$ and $n$ the largest number among all $k \in \mathbb{N} \cup$ $\{\infty\}$ such that $\mathscr{D} \subset \mathscr{D}\left(T^{k}\right)$. Then the following statement hold:

(1) Suppose that $\mathscr{D}^{\infty}(T)$ is a core for all $T^{k}, k \in \mathbb{N}$. Then

$$
l_{s}\left(T^{[1]}\right)=n \quad \text { and } \mathfrak{M}_{s}\left(T^{[1]}\right)=\mathfrak{P}_{n}\left(T^{[1]}\right),
$$

i.e. $\mathfrak{M}_{s}\left(T^{[1]}\right)$ is regular.

(2) Suppose, in addition, that $\mathscr{D}\left(T^{* k}\right)=\mathscr{D}\left(T^{k *}\right), \forall k \in \mathbb{N}$. Then

$$
\mathfrak{M}_{s}\left(T^{[1]}\right)=\mathfrak{P}_{n}\left(T^{[1]}\right) \text { and } \mathfrak{M}_{w}\left(T^{[1]}\right)=\mathfrak{P}_{l_{w}}\left(T^{[1]}\right) \text {, }
$$

i.e. $\mathfrak{M}_{s}\left(T^{[1]}\right)$ and $\mathfrak{M}_{w}\left(T^{[1]}\right)$ are both regular.

Proof. (1) This follows from Proposition 3.2.

(2) Let $k_{1}, k_{2} \in \mathbb{N}$ with $0 \leq k_{1}, k_{2} \leq l_{w}$. Then it follows from the assumptions (1) and (2) that $T^{\left[k_{1}\right]} \square T^{\left[k_{2}\right]}$ exists if and only if $\mathscr{D} \subset \mathscr{D}\left(\left(T^{k_{1}+k_{2}}\right)^{*}\right)=$ $\mathscr{D}\left(\left(T^{*}\right)^{k_{1}+k_{2}}\right)$, which implies $k_{1}+k_{2} \leq l_{w}$. Therefore $\mathfrak{M}_{w}\left(T^{[1]}\right)$ is regular.

The assumptions of Theorem 3.5 (2) are sufficient for the regularity of the two partial subalgebras, but difficult to prove in practice. The following corollary gives stronger, but easier conditions under which the result holds. In Section 5, Ex. 5.2, we give an example (E.4) where the conditions of Theorem 3.5 (2) hold, but those of the corollary do not.

Corollary 3.6. Suppose that either $T \mathscr{D}^{\infty}(T)$ is a core for all $T^{k}, k \in \mathbb{N}$, or the resolvent set $\rho(T)$ is nonempty, that is, $T$ is self-adjoint or maximal symmetric. Then $\mathfrak{M}_{s}\left(T^{[1]}\right)$ and $\mathfrak{M}_{w}\left(T^{[1]}\right)$ are both regular.

Proof. If $T \mathscr{D}^{\infty}(T)$ is a core for all $T^{k}, k \in \mathbb{N}$, so is $\mathscr{D}^{\infty}(T)$. If $\rho(T) \neq \varnothing$, the same conclusion follows from Theorem 1.9 of [18]. Then, in both cases, the statement follows from Lemma 3.4 (2) and Theorem 3.5. 
Remark 3.7. (1) The first statement of Theorem 3.5 holds true if we assume only that one of the deficiency indices is finite ([18], Theorem 1.9). But the second statement does probably not hold under the sole assumption that $\mathscr{D}^{\infty}(T)$ is a core for $T^{k}, \forall k \in \mathbb{N}$ (or that one of the deficiency indices is finite),

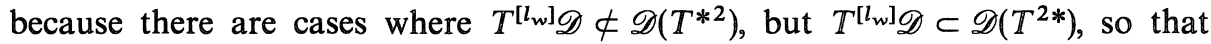
$T^{[2]} \square T^{\left[l_{w}\right]}$ exists and thus $\mathfrak{M}_{w}\left(T^{[1]}\right)$ is not regular.

(2) Let $S$ be the maximal symmetric extension of $T$ and $i \in \rho(S)$. Then the following conditions are easily shown to be equivalent: (i) $T=S$, i.e. $T$ is maximal symmetric; (ii) $(T-i 1) \mathscr{D}^{\infty}(T)$ is a core for all $T^{k}, k \in \mathbb{N}$; (iii) $(T-i 1)$ $\mathscr{D}^{\infty}(T)$ is dense in $\mathscr{H}$ (one shows (i) $\Rightarrow($ ii) $\Rightarrow($ iii) $\Rightarrow(\mathrm{i})$ ). However it is not clear that $T$ being maximal symmetric implies that $T \mathscr{D}^{\infty}(T)$ is a core for all $T^{k}$, $k \in \mathbb{N}$, or vice versa. In other words, the two conditions of Corollary 3.6 seem mutually independent.

In the special case $\mathscr{D}=\mathscr{D}\left(T^{n}\right)$, the situation gets simpler.

Corollary 3.8. Assume $\mathscr{D}=\mathscr{D}\left(T^{n}\right)$. Then:

(1) If $\mathscr{D}\left(T^{n}\right)$ is a core for every $T^{k}(1 \leq k \leq n)$, then

$$
l_{s}\left(T \uparrow \mathscr{D}\left(T^{n}\right)\right)=n \quad \text { and } \quad \mathfrak{M}_{s}\left(T \nmid \mathscr{D}\left(T^{n}\right)\right)=\mathfrak{R}_{s}\left(T \nmid \mathscr{D}\left(T^{n}\right)\right)=\mathfrak{P}_{n}\left(T \nmid \mathscr{D}\left(T^{n}\right)\right) \text {. }
$$

(2) If $T$ is maximal symmetric or $T \mathscr{D}^{\infty}(T)$ is a core for all $T^{k}, k \in \mathbb{N}$, then

$$
l_{s}\left(T \nmid \mathscr{D}\left(T^{n}\right)\right)=l_{w}\left(T \nmid \mathscr{D}\left(T^{n}\right)\right)=n
$$

and

$$
\mathfrak{M}_{s}\left(T \uparrow \mathscr{D}\left(T^{n}\right)\right)=\mathfrak{M}_{w}\left(T \uparrow \mathscr{D}\left(T^{n}\right)\right)=\mathfrak{B}_{n}\left(T \uparrow \mathscr{D}\left(T^{n}\right)\right)
$$

for every $n \in \mathbb{N}$. In particular, if $T$ is self-adjoint, then $\mathfrak{B}_{n}\left(T \uparrow \mathscr{D}\left(T^{n}\right)\right)$ is a standard, abelian partial $O^{*}$-algebra on $\mathscr{D}\left(T^{n}\right)$.

Proof. (1) We must have $\mathscr{D}\left(T^{n+1}\right) \neq \mathscr{D}\left(T^{n}\right)$. Suppose indeed that $\mathscr{D}\left(T^{n}\right)=$ $\mathscr{D}\left(T^{n+1}\right)$. Then, by the closed graph theorem, the norms $\|\cdot\|_{T^{n}}$ and $\|\cdot\|_{T^{n+1}}$ are equivalent, which, by [3], Proposition 2.1.11, implies that $T$ is bounded. Then the statement (1) follows from Proposition 3.2.

(2) By Theorem 3.5, it is sufficient to show that $l_{w}=n$. Suppose that $l_{w}>n$. Then we have

$$
\mathscr{D}\left(T^{n}\right)=\mathscr{D}\left(T^{*} T^{n}\right)
$$

Given any $x \in \mathscr{H}$, we put

$$
f(\xi)=\left(T^{n} \xi \mid x\right), \quad \xi \in \mathscr{D}\left(T^{n}\right) .
$$

Thus $f$ is a continuous linear functional on the Hilbert space $\left(\mathscr{D}\left(T^{n}\right),\langle. \mid\rangle.\right)$. Hence, by the Riesz theorem, there exists $\eta \in \mathscr{D}\left(T^{n}\right)$ such that

$$
f(\xi)=\langle\xi \mid \eta\rangle, \quad \forall \xi \in \mathscr{D}\left(T^{n}\right),
$$


that is,

$$
\left(T^{n} \xi \mid x\right)=(\xi \mid \eta)+\left(T^{n} \xi \mid T^{n} \eta\right),
$$

which implies by Lemma 3.4 that

$$
x-T^{n} \eta \in \mathscr{D}\left(T^{n *}\right)=\mathscr{D}\left(T^{* n}\right) \subset \mathscr{D}\left(T^{*}\right),
$$

and so by (3.5), $x=\left(x-T^{n} \eta\right)+T^{n} \eta \in \mathscr{D}\left(T^{*}\right)$. Hence $\mathscr{D}\left(T^{*}\right)=\mathscr{H}$. By the closed graph theorem, $T^{*}$ is bounded, and so is $T$, which is a contradiction.

Remark 3.9. If $\mathscr{D}\left(T^{n *}\right) \notin \mathscr{D}\left(T^{*}\right)$, we cannot prove that $\mathscr{D}\left(T^{n}\right) \neq \mathscr{D}\left(T^{*} T^{n}\right)$, hence we cannot determine the structure of $\mathfrak{M}_{w}\left(T\left\lceil\mathscr{D}\left(T^{n}\right)\right)\right.$ in general.

Corollary 3.10. (1) Suppose that $T$ is maximal symmetric or that $T \mathscr{D}^{\infty}(T)$ is a core for all $T^{k}, k \in \mathbb{N}$. Let $n, m \in \mathbb{N}, \mathscr{D}=\mathscr{D}\left(T^{m *} T^{n}\right)$ and $T^{[1]}=T \uparrow \mathscr{D}$. Then

$$
n \leq 1_{s}\left(T^{[1]}\right) \leq m+n=l_{w}\left(T^{[1]}\right),
$$

and

$$
\mathfrak{P}_{n}\left(T^{[1]}\right) \subset \mathfrak{M}_{s}\left(T^{[1]}\right)=\mathfrak{P}_{l_{s}\left(T^{[1]}\right)}\left(T^{[1]}\right) \subset \mathfrak{M}_{w}\left(T^{[1]}\right)=\mathfrak{P}_{n+m}\left(T^{[1]}\right) .
$$

(2) In particular, when $m=1$ and $T$ is maximal symmetric (hence $\rho(T) \neq$ $\emptyset)$, but not self-adjoint,

$$
l_{s}\left(T^{[1]}\right)=n \quad \text { and } \mathfrak{M}_{s}\left(T^{[1]}\right)=\mathfrak{P}_{n}\left(T^{[1]}\right) .
$$

Proof. (1) First we can prove, exactly as in Lemma 3.4, that

$$
\mathscr{D}\left(T^{m *} T^{n}\right)=\mathscr{D}\left(T^{* m} T^{n}\right) .
$$

Then, by Theorem 3.5, it is sufficient to show that $l_{w}\left(T^{[1]}\right)=m+n$. Clearly $m+n \leq l_{w}\left(T^{[1]}\right)$. Suppose that $m+n<l_{w}\left(T^{[1]}\right)$. This means

$$
\mathscr{D}\left(T^{* m} T^{n}\right)=\mathscr{D}\left(T^{* m+1} T^{n}\right) .
$$

Exactly as in the proof of Corollary 3.8 (2), we can show that for each $x \in$ $\mathscr{H}$ there exists an element $\eta$ of $\mathscr{D}\left(T^{m+n}\right)$ such that $x-T^{m+n} \eta \in \mathscr{D}\left(\left(T^{m+n}\right)^{*}\right)=$ $\mathscr{D}\left(T^{* m+n}\right) \subset \mathscr{D}\left(T^{*}\right)$. On the other hand, $T^{m+n} \eta=T^{* m} T^{n} \eta \in \mathscr{D}\left(T^{*}\right)$, by (3.6), and so,

$$
x=\left(x-T^{m+n} \eta\right)+T^{m+n} \eta \in \mathscr{D}\left(T^{*}\right) .
$$

Hence $\mathscr{D}\left(T^{*}\right)=\mathscr{H}$. By the closed graph theorem, $T$ is bounded, which is a contradiction.

(2) Let $m=1$. Suppose that $\mathscr{D} \subset \mathscr{D}\left(T^{n+1}\right)$. Then we have

$$
\mathscr{D}\left(\left(T-r_{0} 1\right)^{*}\left(T-r_{0} 1\right)^{n}\right) \subset \mathscr{D}\left(\left(T-r_{0} 1\right)^{n+1}\right), r_{0} \in \rho(T) .
$$


It follows that $\mathscr{D}\left(\left(T-r_{0} 1\right)^{*}\right) \subset \mathscr{D}\left(\left(T-r_{0} 1\right)\right)$, which contradicts the fact that $T$ is not self-adjoint. Hence, $n$ is the largest among all $k \in \mathbb{N} \cup\{\infty\}$ such that $\mathscr{D} \subset \mathscr{D}\left(T^{k}\right)$, and thus it follows from Theorem 3.5 that $l_{s}\left(T^{[1]}\right)=n$ and $\mathfrak{M}_{s}\left(T^{[1]}\right)=\mathfrak{P}_{n}\left(T^{[1]}\right)$. The remaining assertions are proved as in Theorem 3.5.

Remark 3.11. Let $\mathscr{D}=\mathscr{D}\left(T^{m *} T^{n}\right)$. Then $l_{s}\left(T^{[1]}\right)=n$ if and only if $n$ is the largest among all $k \in \mathbb{N} \cup\{\infty\}$ such that $\mathscr{D} \subset \mathscr{D}\left(T^{k}\right)$, which happens if and only if $\mathscr{D}\left(\left(T-r_{0} 1\right)^{* m}\right) \not \subset \mathscr{D}\left(\left(T-r_{0} 1\right)\right)$. Thus, when $m \geq 2$, we don't know whether $l_{s}\left(T^{[1]}\right)=n$. On the other hand, without the assumption $\rho(T) \neq \varnothing$, $\mathfrak{M}_{w}\left(T^{[1]}\right)$ need not be regular (see Example 5.3 below).

\section{§4. Standardness of $\mathfrak{D R}_{s}\left(T^{[1]}\right)$ and $\mathfrak{D R}_{w}\left(T^{[1]}\right)$}

In this Section, we shall investigate the question of standardness of the two partial $O^{*}$-algebras $\mathfrak{M}_{s}\left(T^{[1]}\right)$ and $\mathfrak{M}_{w}\left(T^{[1]}\right)$. We recall that a partial $O^{*}$ algebra $\mathfrak{M}$ is standard if $X^{+}=X^{*}$ for every $X \in \mathfrak{M}$. As before, $T$ will be a closed symmetric unbounded operator in $\mathscr{H}, \mathscr{D}$ a core for $T, T^{[1]}=T \uparrow \mathscr{D}$ and $n$ the largest number among all $k \in \mathbb{N} \cup\{\infty\}$ such that $\mathscr{D} \subset \mathscr{D}\left(T^{k}\right)$. In the case of an $O^{*}$-algebra, the following results have been obtained in [19], Theorem 2.1:

Proposition 4.1. Suppose that $T^{[1]}=T \uparrow \mathscr{D} \in \mathscr{L}^{+}(\mathscr{D})$. Then the following statements are equivalent.

(1) The closure of $\mathfrak{P}\left(T^{[1]}\right)$ is standard.

(2) The closure of $\mathfrak{P}\left(T^{[1]}\right)$ is self-adjoint.

(3) $\mathfrak{P}\left(T^{[1]}\right)_{w}^{\prime} \hat{\mathscr{D}}\left(\mathfrak{P}\left(T^{[1]}\right)\right) \subset \hat{\mathscr{D}}\left(\mathfrak{P}\left(T^{[1]}\right)\right)$.

(4) $T$ is self-adjoint and $\hat{\mathscr{D}}\left(\mathfrak{P}\left(T^{[1]}\right)\right)=\bigcap_{k=1}^{\infty} \mathscr{D}\left(T^{k}\right)$.

(5) $T^{[k]}$ is essentially self-adjoint for $k=1,2, \ldots$.

We assume now that $T \mathscr{D} \notin \mathscr{D}$ and extend these results to the partial $O^{*}$ algebras $\mathfrak{M}_{s}\left(T^{[1]}\right)$ and $\mathfrak{M}_{w}\left(T^{[1]}\right)$.

For convenience, we recall that the weak bounded commutant of a ${ }^{+}$-invariant subset $\mathfrak{N}$ of $\mathscr{L}^{+}(\mathscr{D}, \mathscr{H})$ is defined $[7,20]$ as

$$
\mathfrak{N}_{w}^{\prime}=\left\{C \in \mathscr{B}(\mathscr{H}) ;(C X \xi \mid \eta)=\left(C \xi \mid X^{+} \eta\right) \text {, for each } X \in \mathfrak{N} \text { and } \xi, \eta \in \mathscr{D}\right\} \text {. }
$$

Then the commutants $\mathfrak{M}_{s}\left(T^{[1]}\right)_{w}^{\prime}$ and $\mathfrak{M}_{w}\left(T^{[1]}\right)_{w}^{\prime}$ have the following properties.

Lemma 4.2. Let $T$ be a closed symmetric operator and $\mathscr{D}$ a dense subspace of the Hilbert space $\mathscr{D}(T), T^{[1]}=T \uparrow \mathscr{D}$. Then:

(1) $\mathfrak{M}_{s}\left(T^{[1]}\right)_{w}^{\prime}=\{T \uparrow \mathscr{D}\}_{w}^{\prime}$ and it contains the Cayley transform $U$ of $T$.

(2) The following statements are equivalent:

(2.a) $T$ is self-adjoint;

(2.b) $\mathfrak{M}_{s}\left(T^{[1]}\right)_{w}^{\prime}$ is a von Neumann algebra;

(2.c) There exists a von Neumann algebra $\mathfrak{U} \subset \mathfrak{M}_{s}\left(T^{[1]}\right)_{w}^{\prime}$ containing the Cayley transform $U$ of $T$. 
(3) Let $T$ be self-adjoint and $\mathfrak{M}_{w}\left(T^{[1]}\right)$ regular (thus $\mathfrak{M}_{w}\left(T^{[1]}\right)=\mathfrak{P}_{n}\left(T^{[1]}\right)$ ); then

$$
\mathfrak{M}_{s}\left(T^{[1]}\right)_{w}^{\prime}=\mathfrak{M}_{w}\left(T^{[1]}\right)_{w}^{\prime} .
$$

Proof. (1) The equality of the commutants was stated in [6] Proposition 4.7. The Cayley transform $U$ of $T$ commutes with $T$, i.e. $U T \subset T U$, and this implies the statement.

(2) $\bullet(2 . a) \Rightarrow(2 . b)$ : See [6] Proposition 4.7 (2).

- $(2 . b) \Rightarrow(2 . c)$ : Obvious.

- (2.c) $\Rightarrow(2 . a)$ : This can be proved as in [14] Lemma 4.2.

(3) See [6] Corollary 4.8.

Remark 4.3. Even if $\mathfrak{M}_{w}\left(T^{[1]}\right)$ is regular, but $n<l_{w}, \mathfrak{M}_{w}\left(T^{[1]}\right)_{w}^{\prime}$ need not equal $\mathfrak{M}_{s}\left(T^{[1]}\right)_{w}^{\prime}$; also $U \in \mathfrak{M}_{s}\left(T^{[1]}\right)_{w}^{\prime}$, but $U \notin \mathfrak{M}_{w}\left(T^{[1]}\right)_{w}^{\prime}$ in general (see Example 5.2, E.4 below).

Theorem 4.4. The following statements are equivalent:

(1) The full closure $\hat{\mathfrak{M}}_{s}\left(T^{[1]}\right)$ of $\mathfrak{M}_{s}\left(T^{[1]}\right)$ is standard.

(1') The full closure $\hat{\mathfrak{M}}_{w}\left(T^{[1]}\right)$ of $\mathfrak{M}_{w}\left(T^{[1]}\right)$ is standard.

(2) $\hat{\mathfrak{M}}_{s}\left(T^{[1]}\right)$ is self-adjoint.

(2') $\mathfrak{M}_{w}\left(T^{[1]}\right)=\mathfrak{P}_{n}\left(T^{[1]}\right)$ and it is essentially self-adjoint.

(3) $\mathfrak{M}_{s}\left(T^{[1]}\right)_{w}^{\prime} \hat{\mathscr{D}}\left(\mathfrak{M}_{s}\left(T^{[1]}\right)\right) \subset \hat{\mathscr{D}}\left(\mathfrak{M}_{s}\left(T^{[1]}\right)\right)$.

(4) $T$ is self-adjoint and $\hat{\mathscr{D}}\left(\mathfrak{M}_{s}\left(T^{[1]}\right)\right)=\mathscr{D}\left(T^{m}\right)$ for some $m \in \mathbb{N} \cup\{\infty\}$.

(4') $T$ is self-adjoint and $\mathscr{D}\left(\mathfrak{M}_{w}\left(T^{[1]}\right)\right)=\mathscr{D}\left(T^{m}\right)$ for some $m \in \mathbb{N} \cup\{\infty\}$.

(5) $T^{[1]}, T^{[2]} \ldots T^{[n]}$ are essentially self-adjoint.

When this is the case,

$$
\mathfrak{M}_{w}\left(T^{[1]}\right)=\mathfrak{M}_{s}\left(T^{[1]}\right)=\mathfrak{P}_{n}\left(T^{[1]}\right) .
$$

Proof. The equivalence of (1) to (5) follows from [1], Proposition 4.9, except the implication $(3) \Rightarrow(5)$, that we prove now. Let $\hat{\mathscr{D}} \equiv \hat{\mathscr{D}}\left(\mathfrak{M}_{s}\left(T^{[1]}\right)\right)$. Suppose that $\mathfrak{M}_{s}\left(T^{[1]}\right)_{w}^{\prime} \hat{\mathscr{D}} \subset \hat{\mathscr{D}}$. Since $\hat{\mathfrak{M}}_{s}\left(T^{[1]}\right)_{w}^{\prime}=\mathfrak{M}_{s}\left(T^{[1]}\right)_{w}^{\prime}[1]$, it follows that $\mathfrak{M}_{s}\left(T^{[1]}\right)_{w}^{\prime}$ is a von Neumann algebra. By Lemma $4.2, T$ is self-adjoint and therefore $l_{w}=n$. If $n=1$, the statement is clear. Let $n \geq 2$ and define $T=$ $\int_{-\infty}^{\infty} \lambda d E(\lambda)$ and $E_{m}=\int_{-m}^{m} d E(\lambda), m \in \mathbb{N}$. Since $E_{m} \in\left\{T^{[1]}\right\}_{w}^{\prime}=\mathfrak{M}_{s}\left(T^{[1]}\right)_{w}^{\prime}$, we get $E_{m} \hat{\mathscr{D}} \subset \hat{\mathscr{D}}, \forall m \in \mathbb{N}$, and therefore $\bigcup_{m \in \mathbb{N}} E_{m} \hat{\mathscr{D}} \subset \hat{\mathscr{D}}$. Since $T^{[2]} \in \mathfrak{M}_{s}\left(T^{[1]}\right)$, it follows that $\mathscr{D} \subset \mathscr{D}\left(T^{2}\right)$ and $\bigcup_{m \in \mathbb{N}} E_{m} \hat{\mathscr{D}}$ is dense in $\bigcup_{m \in \mathbb{N}} E_{m} \mathscr{D}\left(T^{2}\right)$ with respect to the Hilbert norm $\|\cdot\|_{T^{2}}$. Furthermore, $\bigcup_{m \in \mathbb{N}} E_{m} \mathscr{D}\left(T^{2}\right)$ is dense in the Hilbert space $\mathscr{D}\left(T^{2}\right)$. Hence, $\hat{\mathscr{D}}$ is a core for $T^{2}$. On the other hand,

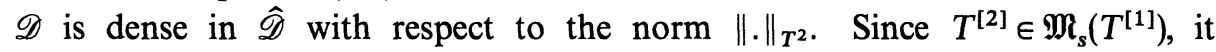
follows that $\mathscr{D}$ also is a core for $T^{2}$. Repeating this argument, we can show that $T^{[1]}, T^{[2]} \ldots T^{[n]}$ are essentially self-adjoint. This completes the proof. 
Remark 4.5. (1) Even if $T$ is self-adjoint and $\mathfrak{M}_{s}\left(T^{[1]}\right)=\mathfrak{M}_{w}\left(T^{[1]}\right)=$ $\mathfrak{P}_{n}\left(T^{[1]}\right), T^{[1]}, T^{[2]} \ldots T^{[n]}$ are not necessarily essentially self-adjoint (see Example 5.1 below).

(2) Contrary to the case of $\mathfrak{M}_{s}\left(T^{[1]}\right)$, even if $\mathfrak{M}_{w}\left(T^{[1]}\right)$ is self-adjoint (and therefore $\left.\mathfrak{M}_{w}\left(T^{[1]}\right)_{w}^{\prime} \hat{\mathscr{D}}\left(\mathfrak{M}_{w}\left(T^{[1]}\right)\right) \subset \hat{\mathscr{D}}\left(\mathfrak{M}_{w}\left(T^{[1]}\right)\right)\right), T^{[1]}, T^{[2]} \ldots T^{[n]}$ need not be essentially self-adjoint (see Example 5.2, E.4 below).

\section{§5. Examples}

Example 5.1. Let $T$ be an arbitrary unbounded self-adjoint operator in $\mathscr{H}$ and take any $n \in \mathbb{N}$. As shown by Schmüdgen ([18], Theorem 5.3), there exists a subspace $\mathscr{D}$ of $\mathscr{D}\left(T^{n}\right)$ such that $\mathscr{D}$ is a core for $T^{n-1}, T^{[n]}=T^{n} \uparrow \mathscr{D}$ is a closed symmetric operator in $\mathscr{H}$ with infinite deficiency indices and $\mathscr{D} \cap$ $\mathscr{D}\left(T^{n+1}\right)=\{0\}$. Then we have:

$$
l_{s}\left(T^{[1]}\right)=l_{w}\left(T^{[1]}\right)=n \quad \text { and } \quad \mathfrak{M}_{s}\left(T^{[1]}\right)=\mathfrak{M}_{w}\left(T^{[1]}\right)=\mathfrak{B}_{n}\left(T^{[1]}\right),
$$

but the latter is not standard, because $T^{n} \uparrow \mathscr{D}$ is not self-adjoint.

$(\mathbb{E} .2) \quad \hat{\mathscr{D}}\left(\mathfrak{M}_{s}\left(T^{[1]}\right)\right)=\hat{\mathscr{D}}\left(\mathfrak{M}_{w}\left(T^{[1]}\right)\right)=\mathscr{D} \subsetneq \mathscr{D}\left(T^{n}\right)=\mathscr{D}^{*}\left(\mathfrak{M}_{w}\left(T^{[1]}\right)\right)=\mathscr{D}^{*}\left(\mathfrak{M}_{s}\left(T^{[1]}\right)\right)$

and thus $\imath^{*}\left(\mathfrak{M}_{w}\left(T^{[1]}\right)\right)=\iota^{*}\left(\mathfrak{M}_{s}\left(T^{[1]}\right)\right)=\mathfrak{B}_{n}\left(T \uparrow \mathscr{D}\left(T^{n}\right)\right)$, and this is a standard partial $O^{*}$-algebra on $\mathscr{D}\left(T^{n}\right)$.

Example 5.2. As in [1], let us consider the closed operators $S, T$ and $H$ in the Hilbert space $\mathbb{L}^{2}[0,1]$ defined by

$$
\begin{aligned}
. \mathscr{D}(S) & =\left\{f \in C[0,1]: f(x)-f(0)=\int_{0}^{x} f_{1}(t) d t \text { for some } f_{1} \in L^{2}[0,1]\right\} \\
S f & =-i f_{1}, f \in \mathscr{D}(S) ; \\
. \mathscr{D}(T) & =\{f \in \mathscr{D}(S): f(1)=f(0)=0\}, \\
T & =S \uparrow \mathscr{D}(T) ; \\
\mathscr{D}(H) & =\{f \in \mathscr{D}(S): f(1)=f(0)\}, \\
H & =S \uparrow \mathscr{D}(H) .
\end{aligned}
$$

As it is well-known, $T^{*}=S$ and $H$ is self-adjoint. It is possible to describe explicitly the partial *-algebras generated by the above operators on the following domains:

$$
\begin{aligned}
& \mathscr{D}_{0}^{(n)}=\left\{f \in C^{(n)}[0,1] ; f^{(k)}(0)=f^{(k)}(1)=0, k=0,1,2, \ldots n\right\}, \\
& \mathscr{D}_{\square}^{(n)}=\left\{f \in C^{(n)}[0,1] ; f \in \mathscr{D}_{0}^{(n-1)} \text { and } f^{(n)} \in \mathscr{D}(S)\right\}, \\
& \mathscr{D}^{(n)}=\left\{f \in C^{(n)}[0,1] ; f^{(k)}(0)=f^{(k)}(1), k=0,1,2, \ldots n\right\},
\end{aligned}
$$

for $n \in \mathbb{N} \cup\{\infty\}$. Then we have 
(1) For each $n \in \mathbb{N}, T^{n}$ is a closed symmetric operator in $L^{2}[0,1]$ with domain

$$
\mathscr{D}\left(T^{n}\right)=\left\{f \in \mathscr{D}_{0}^{(n-1)} ; f^{(n-1)} \in \mathscr{D}(T)\right\} \supset \mathscr{D}_{0}^{(n)} ;
$$

in particular, $\mathscr{D}_{0}^{(\infty)}=\mathscr{D}^{\infty}(T)$. Moreover, $\mathscr{D}_{0}^{(\infty)}$ is a core for $T^{n}, \forall n \in \mathbb{N}$.

(2) $\mathscr{D}_{0}^{(n)} \subset \mathscr{D}_{\square}^{(n)} \subset \mathscr{D}\left(T^{n}\right)$, but $\mathscr{D}_{\square}^{(n)} \not \subset \mathscr{D}\left(T^{n+1}\right)$.

Moreover, $\mathscr{D}_{\square}^{(n)}$ is a core for each $T^{k}, 1 \leq k \leq n$.

(3) $\mathscr{D}\left(H^{n}\right)=\left\{f \in \mathscr{D}^{(n-1)} ; f^{(n-1)} \in \mathscr{D}(H)\right\} \supset \mathscr{D}^{(n)}$, for each $n \in \mathbb{N} . \mathscr{D}^{(\infty)}=\mathscr{D}^{\infty}(H)$ and $H^{n} \uparrow \mathscr{D}^{(\infty)}$ is essentially self-adjoint for each $n \in \mathbb{N}$.

Taking into account these statements and the previous propositions, we get the following results:

$$
\begin{aligned}
& \mathfrak{M}_{s}\left(T \nmid \mathscr{D}_{0}^{(n)}\right)=\mathfrak{M}_{w}\left(T \nmid \mathscr{D}_{0}^{(n)}\right)=\mathfrak{P}_{n}\left(T \nmid \mathscr{D}_{0}^{(n)}\right), n \in \mathbb{N}, \\
& \mathfrak{M}_{s}\left(T \uparrow \mathscr{D}_{0}^{(\infty)}\right)=\mathfrak{M}_{w}\left(T \nmid \mathscr{D}_{0}^{(\infty)}\right)=\mathfrak{P}\left(T \uparrow \mathscr{D}_{0}^{(\infty)}\right),
\end{aligned}
$$

and the latter is a closed $O^{*}$-algebra on $\mathscr{D}_{0}^{(\infty)}$.

$$
\begin{aligned}
\mathfrak{M}_{s}\left(T \uparrow \mathscr{D}_{\square}^{(n)}\right) & =\mathfrak{P}_{n}\left(T \nmid \mathscr{D}_{\square}^{(n)}\right) \\
& \subsetneq \mathfrak{M}_{w}\left(T \uparrow \mathscr{D}_{\square}^{(n)}\right)=\left\{\sum_{k=0}^{n} \alpha_{k} T^{k} \uparrow \mathscr{D}_{\square}^{(n)}+\alpha_{n+1} T^{*} T^{n} \uparrow \mathscr{D}_{\square}^{(n)} ;\right. \\
& \left.\alpha_{k} \in \mathbb{C}, k=0,1,2, \ldots, n+1\right\} .
\end{aligned}
$$

This is an example where the assumptions of Theorem 3.5 (2) hold, but not those of Corollary 3.6. Indeed, the defect indices of $T$ are $(1,1)$, so that $\mathscr{D}^{\infty}(T)$ is a core for all $T^{k}, k \in \mathbb{N}$, but $T \mathscr{D}^{\infty}(T)$ is not (for instance, $T \mathscr{D}^{\infty}(T)$ is not a core for $T)$. On the other hand, one has $\mathscr{D}\left(T^{* k}\right)=\mathscr{D}\left(T^{k *}\right), \forall k \in \mathbb{N}$, and thus Theorem 3.5 applies: $\mathfrak{M}_{s}\left(T \uparrow \mathscr{D}_{\square}^{(n)}\right)$ and $\mathfrak{M}_{w}\left(T \nmid \mathscr{D}_{\square}^{(n)}\right)$ are both regular.

Furthermore, $T \uparrow \mathscr{D}_{\square}^{(1)}$ is not essentially self-adjoint, but $\mathfrak{M}_{w}\left(T \uparrow \mathscr{D}_{\square}^{(1)}\right)$ is a self-adjoint partial $O^{*}$-algebra on $\mathscr{D}_{\square}^{(1)}$, and thus

$$
\mathfrak{M}_{w}\left(T \nmid \mathscr{D}_{\square}^{(1)}\right)_{w}^{\prime} \varsubsetneqq\left\{T \uparrow \mathscr{D}_{\square}^{(1)}\right\}_{w}^{\prime}=\mathfrak{M}_{s}\left(T \uparrow \mathscr{D}_{\square}^{(1)}\right)_{w}^{\prime} .
$$

In fact, it is easily seen that $\mathscr{D}_{\square}^{(1)}=\mathscr{D}\left(T^{*} T\right)$ and $\mathfrak{M}_{w}\left(T \nmid \mathscr{D}_{\square}^{(1)}\right)=\left\{\alpha_{0} 1+\alpha_{1} T \nmid \mathscr{D}_{0}^{(1)}+\right.$ $\left.\alpha_{2} T^{*} T \uparrow \mathscr{D}_{0}^{(1)} ; \alpha_{i} \in \mathbb{C}\right\}$. Since

$$
\begin{aligned}
\mathscr{D}^{*}\left(\mathfrak{M}_{w}\left(T \uparrow \mathscr{D}_{\square}^{(1)}\right)\right. & =\mathscr{D}\left(\left(T \uparrow \mathscr{D}_{\square}^{(1)}\right)^{*}\right) \cap \mathscr{D}\left(\left(T^{*} T \uparrow \mathscr{D}_{\square}^{(1)}\right)^{*}\right) \\
& =\mathscr{D}\left(T^{*}\right) \cap \mathscr{D}\left(T^{*} T\right)=\mathscr{D}\left(T^{*} T\right)=\mathscr{D}_{\square}^{(1)},
\end{aligned}
$$

it follows that $\mathfrak{M}_{w}\left(T \nmid \mathscr{D}_{\square}^{(1)}\right)$ is self-adjoint, so that $\mathfrak{M}_{w}\left(T \uparrow \mathscr{D}_{\square}^{(1)}\right)_{w}^{\prime}$ is a von Neumann algebra. On the other hand, since $T \uparrow \mathscr{D}_{\square}^{(1)}$ is not essentially self-adjoint, it follows from Lemma 4.2 that $\mathfrak{M}_{s}\left(T \uparrow \mathscr{D}_{\square}^{(1)}\right)_{w}^{\prime}$ is not a von Neumann algebra. Therefore, $\mathfrak{M}_{w}\left(T \nmid \mathscr{D}_{\square}^{(1)}\right)_{w}^{\prime} \subsetneq \mathfrak{M}_{s}\left(T \nmid \mathscr{D}_{\square}^{(1)}\right)_{w}^{\prime}$. 
(E.5) $\mathfrak{M}_{s}\left(H \uparrow \mathscr{D}^{(n)}\right)=\mathfrak{M}_{w}\left(H\left\lceil\mathscr{D}^{(n)}\right)=\mathfrak{P}_{n}\left(H \uparrow \mathscr{D}^{(n)}\right)\right.$, and its full closure is a standard partial $O^{*}$-algebra $\mathfrak{P}_{n}\left(H \mid \mathscr{D}\left(H^{n}\right)\right)$; in particular,

$$
\mathfrak{M}_{s}\left(H \uparrow \mathscr{D}^{(\infty)}\right)=\mathfrak{M}_{w}\left(H \uparrow \mathscr{D}^{(\infty)}\right)=\mathfrak{P}\left(H \uparrow \mathscr{D}^{(\infty)}\right),
$$

and this is a standard partial $O^{*}$-algebra on $\mathscr{D}^{(\infty)}$.

Example 5.3. Let $T$ and $H$ be as in Example 5.2.

(1) Let $\mathscr{D}=\mathscr{D}\left(T^{m *} T^{n}\right)$. Then we have:

(E.6) $l_{s}\left(T^{[1]}\right)=n$ and $\mathfrak{M}_{s}\left(T^{[1]}\right)=\mathfrak{P}_{n}\left(T^{[1]}\right)$.

Furthermore we have

(E.7) When $m \leq n+1$,

$$
l_{w}\left(T^{[1]}\right)=m+n \quad \text { and } \quad \mathfrak{M}_{w}\left(T^{[1]}\right)=\mathfrak{P}_{m+n}\left(T^{[1]}\right) ;
$$

(E.8) When $m>n+1$,

$$
\begin{aligned}
& l_{w}\left(T^{[1]}\right)=2 n+1 \text { and } \mathfrak{R}_{w}\left(T^{[1]}\right)=\mathfrak{P}_{2 n+1}\left(T^{[1]}\right), \text { and } \\
& \left\{T^{[2 n+2]}, \ldots, T^{[m+n]}\right\} \subset \mathcal{S}_{w}\left(T^{[1]}\right) .
\end{aligned}
$$

In this case $\rho\left(T^{[1]}\right)=\varnothing$ and the deficiency indices are both finite.

(2) Let $\mathscr{D}=\mathscr{D}\left(H^{m} T^{n}\right)$. Then we have:

(E.9) $l_{s}\left(T^{[1]}\right)=n<l_{w}\left(T^{[1]}\right)=m+n$, $\mathfrak{M}_{s}\left(T^{[1]}\right)=\mathfrak{P}_{n}\left(T^{[1]}\right) \subset \mathfrak{M}_{w}\left(T^{[1]}\right)=\mathfrak{P}_{n+m}\left(T^{[1]}\right)$.

\section{§6. Final Comments}

Of course, the case examined here is very simple, and the situation becomes already more involved if one considers the partial $O^{*}$-algebras generated by two closed symmetric operators, as is done in [21]. Yet this simple example illustrates clearly the difficulties which are characteristic of partial $O^{*}$-algebras. The lesson we draw from it is twofold. First, most of the pathologies vanish if the basic domain $\mathscr{D}$ is well adapted to the operators one considers (this was already the moral of the earlier work [5]). In physical applications, this remark points of course to the problem of the correct identification of the observables (see also [22] in this respect). On the other hand, assuming the basic operator $T$ to be self-adjoint does not significantly simplify the situation. The second conclusion is that, despite its intricacies, the theory of partial $O^{*}$-algebras offers a number of interesting mathematical challenges. Some of them (e.g. representation theory) are met in our previous work $[1,2,23]$, to which we refer the interested reader. 


\section{Acknowledgments}

Part of this work was performed in the Institut de Physique Théorique, Universite Catholique de Louvain and in the Department of Applied Mathematics, Fukuoka University. We acknowledge gratefully the hospitality of both institutions, and also travel grants from CGRI (Communauté Française de Belgique), Fukuoka University and Università di Palermo, respectively. We all thank G. Epifanio, A. Kargol, F. Mathot and K. Schmüdgen for stimulating discussions about partial $O^{*}$-algebras, and the referee for his constructive suggestions.

\section{References}

[1] Antoine, J.-P., Inoue, A. and Trapani, C., Partial *-algebras of closable operators I. The basic theory and the abelian case, Publ. RIMS, Kyoto Univ., 26 (1990), 359-395.

[2] -, Partial *-algebras of closable operators. II. States and representations of partial *-algebras, Publ. RIMS, Kyoto Univ., 27 (1991), 399-430.

[3] Schmüdgen, K., Unbounded Operator Algebras and Representation Theory, AkademieVerlag, Berlin, 1990.

[4] Antoine, J.-P. and Karwowski, W., Partial *-algebras of closed linear operators in Hilbert space, Publ. RIMS, Kyoto Univ., 21 (1985), 205-236; Add./Err. ibid., 22 (1986), 507-511.

[5] —, Commuting normal operators in partial $O p^{*}$-algebras, Ann. Inst. H. Poincaré, 50 (1989), 125-149.

[6] Antoine, J.-P. and Mathot, F., Partial *-algebras of closed operators and their commutants I. General structure, Ann. Inst. H. Poincaré, 46 (1987), 299-324.

[7] Antoine, J.-P., Mathot, F. and Trapani, C., Partial *-algebras of closed operators and their commutants II. Commutants and bicommutants, Ann. Inst. H. Poincaré, 46 (1987), 325-351.

[8] Epifanio, G. and Trapani, C., $V^{*}$-algebras: a particular class of unbounded operator algebras, J. Math. Phys., 25 (1984), 2633-2637.

[9] Antoine, J.-P., Epifanio, G. and Trapani, C., Complete sets of unbounded observables, Helv. Phys. Acta, 56 (1983), 1175-1186.

[10] Ali, S. T., Stochastic localization, quantum mechanics on phase space and quantum space-time, Rivista Nuovo Cimento, 8 (1985), 1-128.

[11] Davies, E. B., Quantum Theory of Open Systems, Academic Press, London, 1976.

[12] Dubin, D. A., J. Sotelo-Campos, A theory of quantum measurement based on the CCR algebra $L^{+}(\mathscr{W}), \quad Z$. Anal. Anwend., 5 (1986), 1-26.

[13] Ludwig, G., Foundations of Quantum Mechanics, Springer-Verlag, New York, 1983.

[14] Powers, R. T., Self-adjoint algebras of unbounded operators, Commun. Math. Phys., 21 (1971), 85-124.

[15] Lassner, G., Topological algebras of operators, Rep. Math. Phys., 3 (1972), 279-293.

[16] — Topologien auf $O p^{*}$-Algebren, Wiss. Z. Karl-Marx-Univ. Leipzig, Math.-Naturwiss. R., 24 (1975), 465-471.

[17] - Topological algebras and their applications in Quantum Statistics, ibid., 30 (1981), $572-595$.

[18] Schmüdgen, K., On domains of powers of closed symmetric operators, J. Oper. Theory, 9 (1983), 53-75.

[19] Inoue, A. and Takesue, A., Self-adjoint representations of polynomial algebras, Trans. Amer. Math. Soc., 280 (1983), 393-400. 
[20] Borchers, H. J. and Yngvason, J., On the algebra of field operators. The weak commutant and integral decomposition of states, Commun. Math. Phys., 42 (1975), 231-252.

[21] Inoue, A., Partial $O^{*}$-algebras generated by two closed symmetric operators, J. Oper. Theory, to appear.

[22] Dubin, D. A. and Hennings, M. A., Quantum Mechanics, Algebras and Distributions, Pitman Research Notes in Math. Series, Longman, Harlow, 1990.

[23] Antoine, J.-P. and Inoue, A., Representability of invariant positive sesquilinear forms on partial *-algebras, Math. Proc. Camb. Phil. Soc., 108 (1990), 337-353. 